\title{
A Magentoelectric Coefficient Measurement System with a Free-Stress Sample Holder
}

\author{
Jingjing Lu, Xiya Wang, Junhao Wang, Yufei Zhou, Duo Chen, \\ Shibo Wang, Maofa Zhang, Xiangbin Zeng* \\ School of Optical and Electronic Information, Huazhong University of Science and Technology, Wuhan, China \\ Email:*exbzeng@163.com
}

How to cite this paper: Lu, J.J., Wang, X.Y., Wang, J.H., Zhou, Y.F., Chen, D., Wang, S.B., Zhang, M.F. and Zeng, X.B. (2020) A Magentoelectric Coefficient Measurement System with a Free-Stress Sample Holder. Journal of Minerals and Materials Characterization and Engineering, 8, 330-340. https://doi.org/10.4236/jmmce.2020.84020

Received: June 28, 2020

Accepted: July 27, 2020

Published: July 30, 2020

Copyright $\odot 2020$ by author(s) and Scientific Research Publishing Inc. This work is licensed under the Creative Commons Attribution International License (CC BY 4.0).

http://creativecommons.org/licenses/by/4.0/

\begin{abstract}
With less extra stress on the testing sample, a free-stress sample holder was designed to place the sample horizontally. With the free-stress sample holder, a magnetoelectric (ME) coefficient measurement system was developed based on dynamic method. This measurement system has the hardware part and the software part, integrated by the DC magnetic field generation module, the AC magnetic field generation module, the induced $\mathrm{ME}$ voltage detecting module and PC software module. Then, a sample of Terfenol-D/PZT/Terfenol-D trilayer was designed and fabricated, and the relationships of its ME coefficient influenced by the DC magnetic field and the frequency of the AC magnetic field were tested using the measurement system. And the results showed that the free-stress sample holder was proven to improve the accuracy of the measurement system by less stress interference.
\end{abstract}

\section{Keywords}

Magnetoelectric Coefficient, Measurement System, Free-Stress Sample Holder

\section{Introduction}

In magnetoelectric (ME) composites, electric field can control its magnetic properties and magnetic field can control its electric properties. In recent years, the potential application of the ME composites with the considerable ME coefficient at room temperature has attracted wide attention in the memorizers [1] [2], sensors [3] [4] and the MEMS devices [5] [6]. To improve the performance of the ME composite in their applications, the ME coefficient has to be discussed very often.

In the ME composite, the ME effect arises from the magnetostrictive and piezoelectric constituents via interfacial strain [7]. When magnetic field is applied 
on the ME composite, the stress or strain, generated in the magnetostrictive part, passes to the piezoelectric part, in which the induced voltage is generated and detected, and vice versa. Therefore, the ME coefficients is defined by

$\alpha_{\mathrm{E}}=d E / d \sigma \times d \sigma / d H=d E / d H, E, H$ and $\sigma$ are the electric field, the magnetic field and the stress, respectively [8]. The stresses, generated in the magnetostrictive constituents or in the piezoelectric constituents or passed through the interfaces of the two constituents, influence the ME coefficient seriously. Especially, when the ME coefficient is being tested by the ME coefficient measurement system, any extra stress, not from the ME effect, will affect the testing results. It is always the significant problem to design a preferable ME coefficient measurement system for the experimenters.

The stress is the important intermediate quantity of the ME effect, and the impacts of the stress caused by the sample holder on the testing sample cannot be neglected. A little additional stress from the sample holder clamping can increase the error of the testing results, especially it cannot insure the stress from the sample holder clamping always keeping the same value. In this paper, the ME coefficient measurement system was developed to design a free-stress sample holder to avoid additional stress. A sample of Terfenol-D/PZT/Terfenol-D layered composite was tested by the ME coefficient measurement system. Compared with in other clamping ways, the free-stress sample holder is demonstrated to be with less errors.

\section{Theory}

The ME coefficient measurement has two methods, static method [9] and dynamic method [10], in which the latter one is more popular nowadays. The static method uses a DC magnetic field and detects the induced ME voltage, every data of which needs almost half an hour to get the stable data [9]. In the dynamic method, a DC magnetic field and an AC magnetic field are superimposed to get an output ME voltage, which is an AC voltage with the same frequency of the AC magnetic field simultaneously [11] [12] [13]. Many research groups have their homemade ME measurement systems with the dynamic method [14] [15] [16] [17], that often contain DC magnetic field generation module, AC magnetic field generation module, induced ME voltage detecting module and a sample holder. Then, computers and networks are utilized in ME measurement system with the development of computer technology [11] [15] [18]. In this paper, the dynamic method is used with a DC magnetic field $H_{d c}$ and an AC magnetic field $H_{a c}, H_{a c}=h_{0} \sin (\omega t)$, (the total magnetic field $H, H=H_{d c}+h_{0} \sin (w t)$ ), applied to the ME composite layer, and then an induced ME voltage $V_{\text {out }}$ detected in the piezoelectric layer.

The induced ME voltage $V_{\text {out }}$ is a function of the applied magnetic field $H$ and the extra stress $\sigma_{\text {ext }}$ from the clamping ways of the sample holder, as the following equation:

$$
V_{\text {out }}=f(H)+g\left(\sigma_{\text {ext }}\right)
$$


where, $f(H)=$ const $+\alpha H+\beta H^{2}+\gamma H^{3}+\cdots, \alpha, \beta$ and $\gamma$ are the coefficients of the different orders in the $V$-H function; $g\left(\sigma_{\text {ext }}\right)$ is the item related with the extra stress $\sigma_{\text {ext }}$ Regardless of the extra stress $\sigma_{\text {ext }}$, when $h_{0} \ll H_{d c}$, the ME coefficient $\alpha_{E}$ could be calculated by Equation (2),

$$
\alpha_{E}=\frac{\partial E}{\partial H}=\frac{1}{d} \frac{\partial V}{\partial H}=\frac{V_{\text {out }}}{h_{0} d}
$$

where, $d$ is the thickness of the piezoelectric layer.

Considering the extra stress $\sigma_{\text {ext }}$, Equation (2) should be

$$
\alpha_{E}=\frac{V_{o u t}^{\prime}+g_{x} \Delta \sigma}{h_{0} d}=\alpha_{E}^{\prime}+\alpha_{\sigma}=\frac{V_{o u t}}{h_{0} d}
$$

where, $V_{\text {out }}$ is the measurement value of the induced voltage, the item of $V_{\text {out }}^{\prime}$ is caused by the applied magnetic field and The item of $g_{x} \Delta \sigma$ is caused by the extra stress. So $\alpha_{E}^{\prime}$ is the true ME coefficient of the ME effect and $\alpha_{\sigma}$ is the error from the clamping ways of the sample holder. Here, in order to decrease the extra stress $\sigma_{\text {ext }}$ from the clamping ways, a free-stress sample holder is designed to makes the ME coefficient measurement system as accurate as possible.

\section{Measurement System Design}

Based on the dynamic method of ME coefficients, the measurement system is equipped with DC magnetic field generation module, AC magnetic field generation module, the induced ME voltage detecting module, a sample holder and PC software module, shown in Figure 1(a). In the system, the components and their connections are shown in Figure 1(b). PC software can give the instructions to control the output of DC and AC magnetic fields and their signal feedback, process the induced ME voltage signal, calculate and export the ME coefficient data.

\subsection{Magnetic Field Generation Module}

In the measurement system, PC software controls the driven current of the DC source on the electromagnet and tunes the amplitude of $H_{d c}$ Here, HAP30-150 DC power drives PEM5005 type electromagnet to provide a bias magnetic field of $0-4000$ Oe, Simultaneously the electromagnet has a working air gap of 50 $\mathrm{mm}$, where is large enough to place Helmholtz coils. The probe of $\mathrm{CH}-1500$ Gauss meter (the detection range of $0.1 \mathrm{Gs}-30 \mathrm{kGs}$, the accuracy of $0.1 \mathrm{Gs}$ ) is fixed to detect the value of the horizontal magnetic field in the working air gap center of the electromagnet, near the top of the sample.

The DC magnetic field $H_{d c}$ is proportional to the driven current $I, H_{d c}=\mathrm{A} \times I$ $+\mathrm{B}$, where, $\mathrm{A}$ and $\mathrm{B}$ are constants. The relationship of the $\mathrm{DC}$ magnetic field $H_{d c}$ with the current $I$ is shown in Figure 2. It exhibits a good linearity, and matches with the equation $H_{d c}(\mathrm{Oe})=444.7(\mathrm{Oe} / \mathrm{A}) \times I(\mathrm{~A})+22.5(\mathrm{Oe})$. The linear relationship of the bias magnetic field $H_{d c}$ with the current $I$ is written into software to control the DC magnetic field in the next testing, with the error between the calculated value and the measurement value is less than $\pm 0.09 \%$. 
(a) DC magnetic field generation module

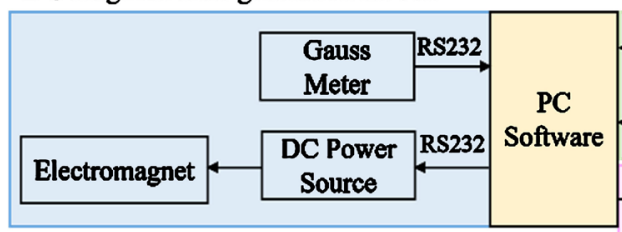
Induced ME voltage detecting module

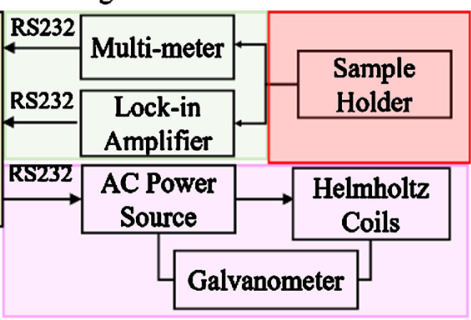

AC magnetic field generation module

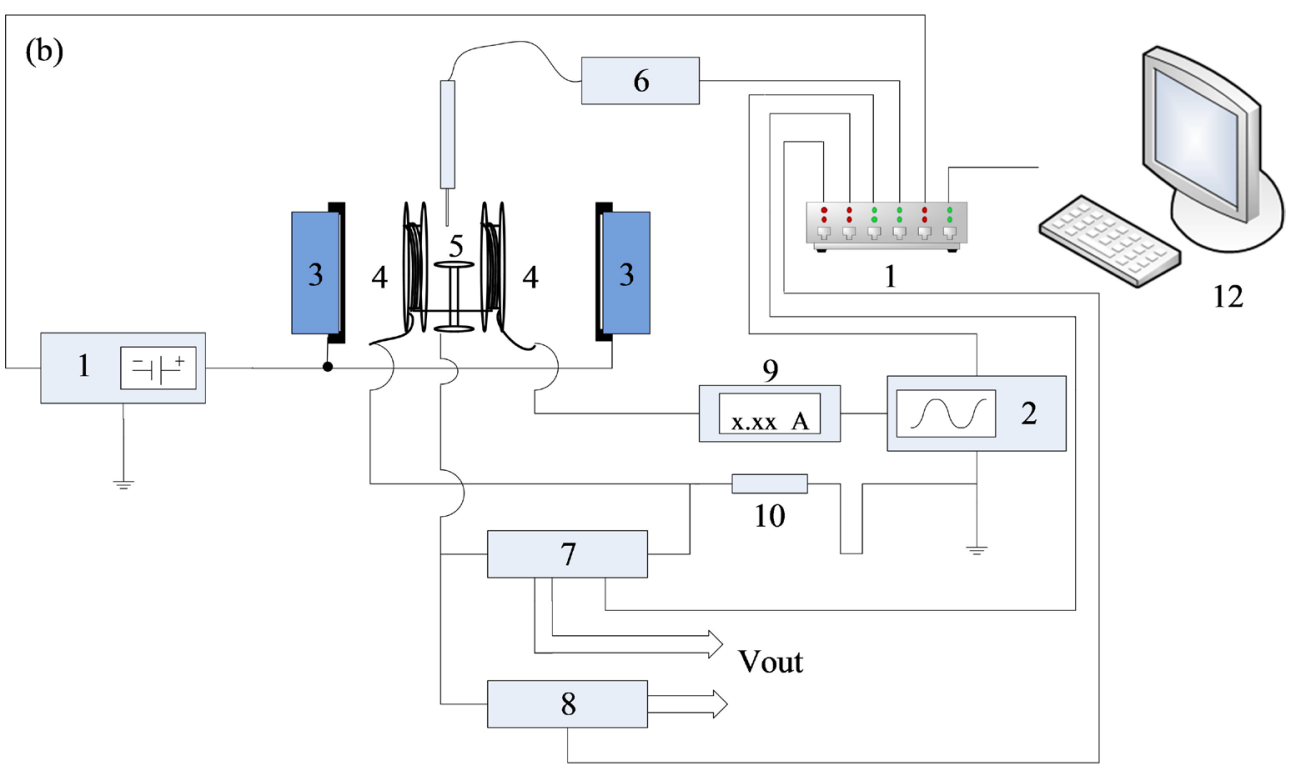

Figure 1. (a) the framework of the ME measurement system; (b) the components of the ME measurement system. (1-DC power source; 2-AC power source; 3-eletromagnet; 4-Helmholtz coils; 5-sample holder; 6-Guass meter; 7-lock-in amplifier; 8-high-precision multi-meter; 9-galvanometer; 10-sampling resistance; 11-USB hub; 12-PC, respectively).

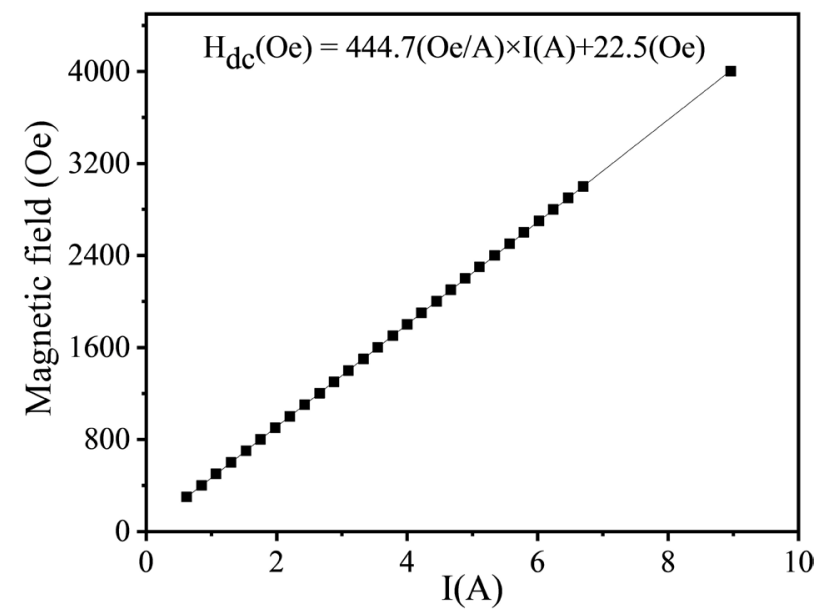

Figure 2. The relationship of the DC magnetic field $H_{d c}$ with the current $I$.

\subsection{AC Magnetic Field Generation Module}

AC source, galvanometer, Helmholtz coils, sampling resistor connect in series to 
generate AC magnetic field in the center of the Helmholtz coils. The AC magnetic field $H_{a c}$ by the Helmholtz coils is proportional to the current in the Helmholtz coils. The current in the circuit, tested by the galvanometer, is used to estimate the AC magnetic field in the center of the Helmholtz coils. The Helmholtz coils are placed in the working air gap between the magnetic poles of the electromagnet and keeping the central axis of the Helmholtz coils coincide with the one of magnetic poles. In order to get a tiny AC magnetic field of $1 \mathrm{Oe}$, the Helmholtz coils are designed with the outside diameter of $80 \mathrm{~mm}$, the inside diameter of $45 \mathrm{~mm}$, the distance between the Helmholtz coils $47 \mathrm{~mm}, 6$ turns of the $1.02 \mathrm{~mm}$-diameter enameled wire and the resistance of $0.4 \Omega$. AC source is the SPF1651 AC source to drive the Helmholtz coils to generate AC magnetic field. The frequency range and its accuracy of the AC magnetic field are 0.01 $300 \mathrm{kHz}$ and $0.1 \mathrm{~Hz}$, respectively. The sampling resistor is $1 \Omega, 10 \mathrm{~W}$. The value and frequency of the AC magnetic field are calibrated by a sinusoidal with 20 turns of the diameter $100 \mathrm{~mm}$, being set into the center of the Helmholtz coils to induce the AC magnetic field $H_{a c}$ generated by the Helmholtz coils. The values of the AC magnetic field under the different current at $1 \mathrm{kHz}$ and at the different frequencies were shown in Figure 3(a) and Figure 3(b). In Figure 3(a), the values of the $\mathrm{AC}$ magnetic field at $1 \mathrm{kHz}$ is linearly related to the current, and 1 Oe alternating magnetic field is achieved when the current is $1 \mathrm{~A}$. In Figure $3(\mathrm{~b})$, at the different frequencies the values of the $\mathrm{AC}$ magnetic field is about 1 Oe with the current $1 \mathrm{~A}$.

\subsection{The Induced ME Voltage Detecting Module}

As the sample size becomes smaller, the fundamental resonance frequency of the sample will increase, that is sometimes over a testing frequency range of one detected device. Here, Lock-in amplifier SR850, with the frequency range of 0.01 $100 \mathrm{kHz}$, and FLUKE8846A multi-meter, with the frequency range of $3-300$ $\mathrm{kHz}$, are used to detect the induced ME voltage together, because the resonance frequency of our sample is over $100 \mathrm{kHz}$. When testing the small voltage output

(a)

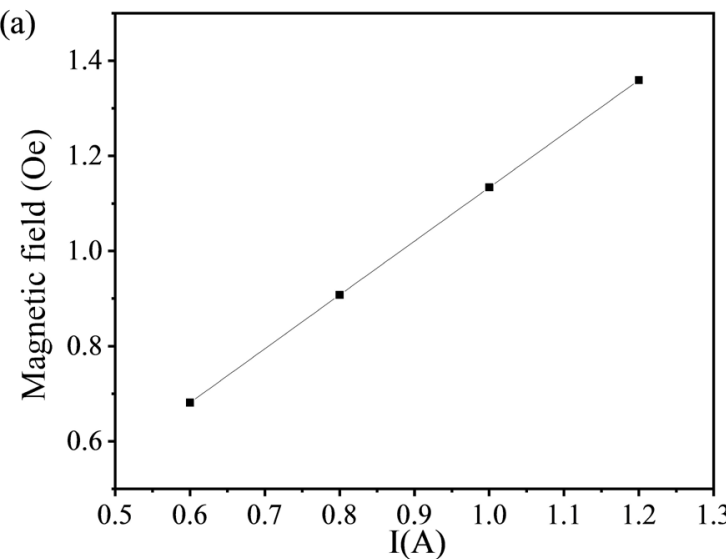

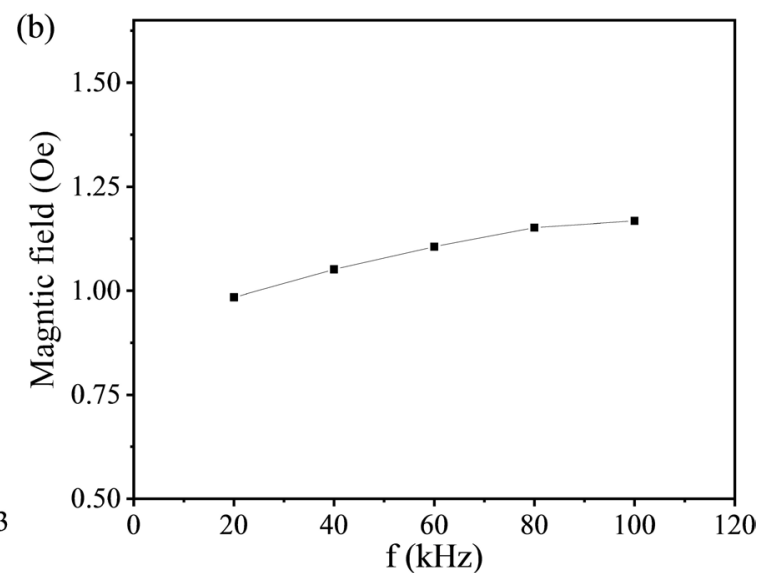

Figure 3. The AC magnetic field (a) with the different current at $1 \mathrm{kHz}$ and (b) at the different frequency, and the inner one is the induced sinusoidal voltage and the current signal in the Helmholtz coils. 
under $100 \mathrm{kHz}$, far below the resonance frequency, the lock-in amplifier works, with the accuracy of $2 \mathrm{nV}$. The voltage output over $100 \mathrm{kHz}$, near the resonance frequency, is detected by the FLUKE8846A multi-meter, with the accuracy of 10 $\mathrm{nV}$.

The performance of noise level of the detected devices was evaluated by testing a PZT layer with the same size of the ME layered composite. The detected voltage signals of the detected devices are shown as Figure 4(a) and Figure 4(b), respectively at $1 \mathrm{kHz}$ as the DC magnetic field of $0-3000$ Oe and at $0.01-300$ $\mathrm{kHz}$ as DC magnetic field of 600 Oe. The amplitude of the noise is about $20 \mathrm{nV}$, which is much smaller than the effective value of the ME induced voltage (several $\mathrm{mV}$ ). The noisy behavior could be related to the environment electromagnetic fluctuation and so on.

\subsection{The Sample Holder}

In the ME coefficient measurement systems, the sample holder always applied a force on the sample vertically [17] or horizontally [19], as shown in Figure 5(a) and Figure 5(b). Either manually or automatically install the sample, it is difficult to keep the extra force constant, which is a source of error about the testing results. In this paper, without extra force on the sample is a way to decrease the influence of the sample holder and the testing error, as in Figure 5(c).

In order to decrease the extra stress from the sample holder clamping, a sample holder is shown in Figure 5, by which little stress is applied on the sample. It

(a)

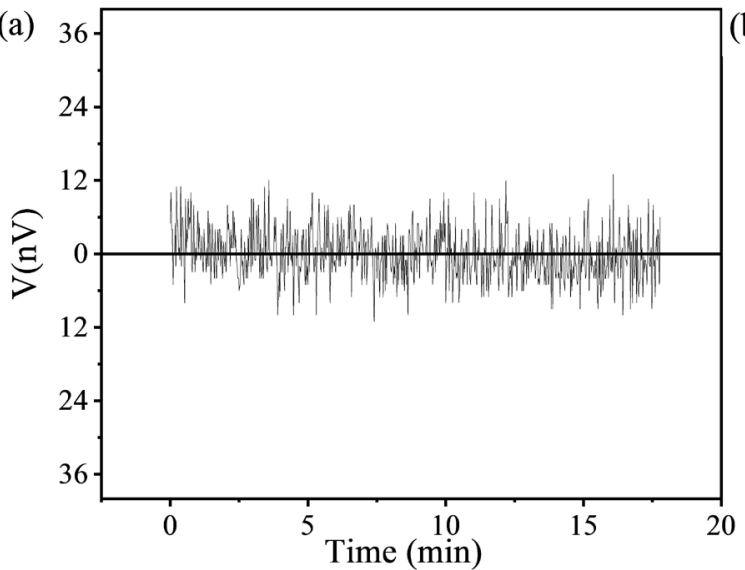

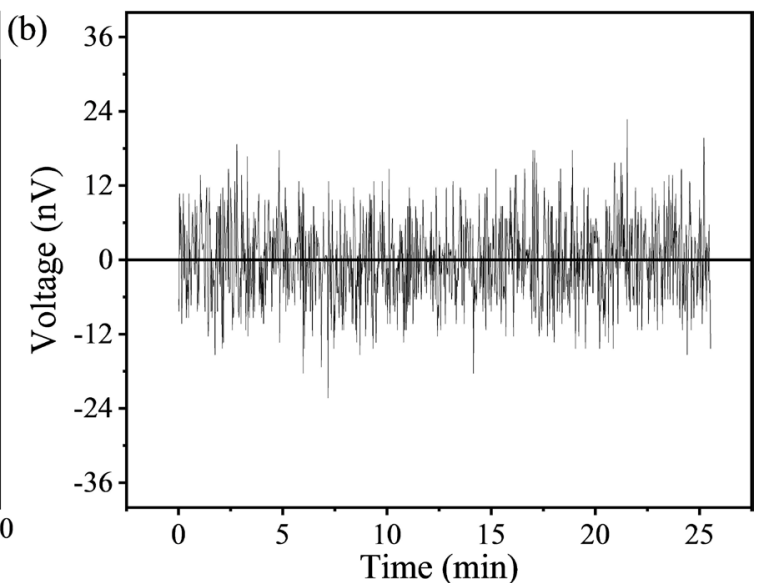

Figure 4. (a) at $1 \mathrm{kHz}$ AC magnetic field, as the DC magnetic field from 0 - 3000 Oe; (b) at 600 Oe DC magnetic field, as the AC magnetic field from $10 \mathrm{~Hz}-200 \mathrm{kHz}$.

(a)

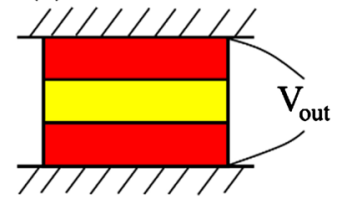

(b)

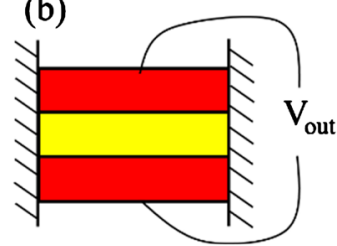

(c)

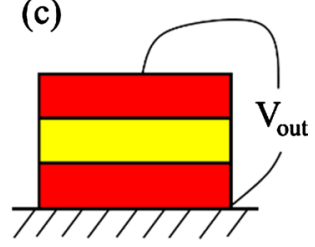

Figure 5. The different clamping ways of the sample holder in ME coefficient measurement system. 
is composed of the pallet, pillar and chassis. A grooving along the pillar is to place the signal line. The sample stage with a recess $16 \times 10 \times 2 \mathrm{~mm}^{3}$ in Figure 6(d) is fit for a PCB board in Figure 6(c). The PCB is embedded in the recess so that a pair of pin header on РСB happens to match a female header on the top of grooving in Figure 6(b). The electrodes of the sample connected to circuit outputs the voltage signal of the piezoelectric layer. The chassis is fixed and the pallet is rotatable with angle scale engraved on the center pillar. By rotating the stage in a horizontal plane angle, the ME coefficient can be tested in the different directions.

\subsection{PC Software Module}

The main working flow chart of PC software is shown in Figure 7. PC software can calculate the ME coefficient data and display them by a graphical form in real time, and then export them by Excel or Txt formats. Two different line chart panels are designed to display the $\alpha-\mathrm{H}_{\mathrm{dc}}$ relationship and the $\alpha-\mathrm{f}_{\mathrm{ac}}$ relationship.

Finally, in the measurement system, all of the instruments are equipped with RS232 interface and PC software controls each device via USB hub.

\section{Test Results}

The ME coefficient of a trilayer composite sample Terfenol-D/PZT/Terfenol-D,

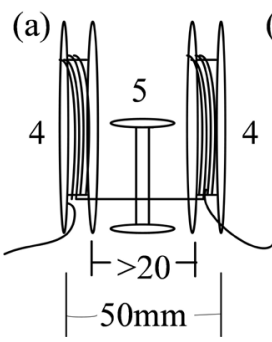

(b)

13

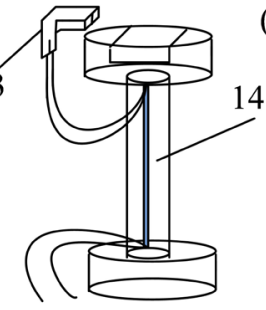

(c)

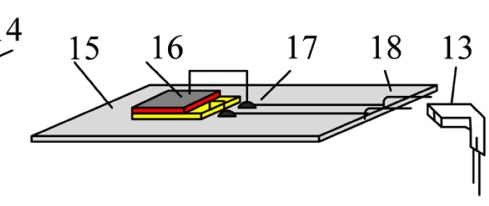

(d)

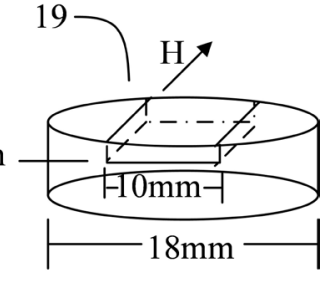

Figure 6. Sample holder. (13-female header; 14-groove for wire; 15-PCB board; 16-electrode; 17-bonding point; 18-pin header; 19-sample stage).
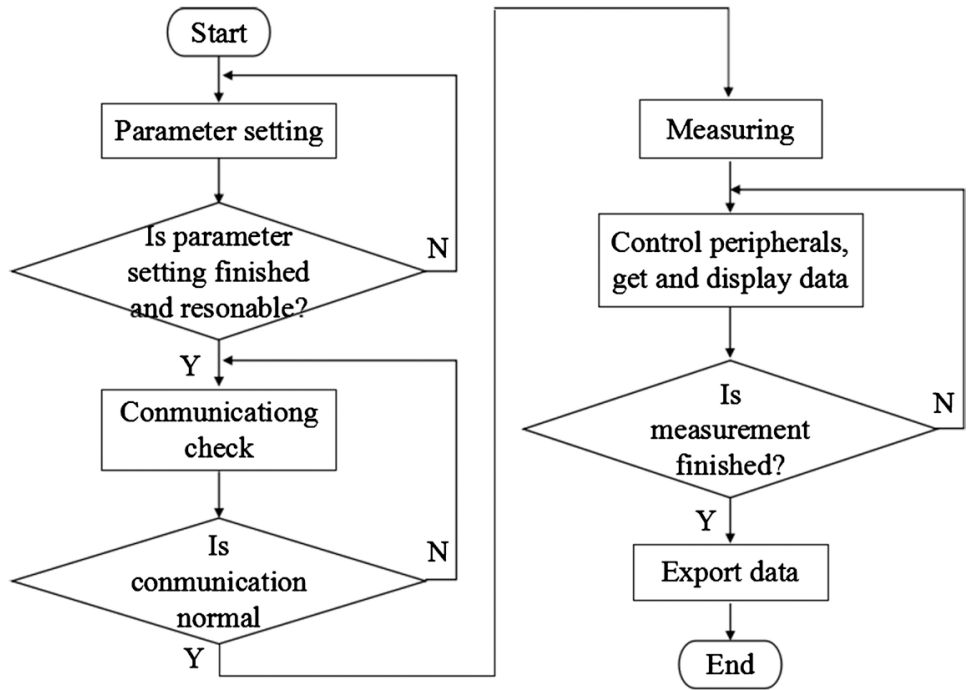

Figure 7. The main program flow chart. 
with the size of $7.6 \times 5.7 \times 0.9 \mathrm{~mm}^{3}$ of Terfenol-D and $7.6 \times 5.7 \times 1.0 \mathrm{~mm}^{3}$ of PZT was tested five times with each clamping ways by the ME coefficient measurement system.

By three holding ways, clamping vertically (Figure 8(a)), clamping horizontally (Figure $8(\mathrm{~b})$ ) and free-stress holding (Figure $8(\mathrm{c})$ ), Figure 8 shows the relationships between the ME coefficient and the DC magnetic field at the AC magnetic field of $1 \mathrm{kHz}$. When $H_{d c}$ is small, the ME coefficient changes rapidly with the DC magnetic field increasing, reaches the maximum ME coefficient near the DC magnetic field of $1100 \mathrm{Oe}$, and then decreases slowly. These experimental results have the same trend with the theoretical and the experimental results in literatures [11]. This phenomenon is due to the dynamic magnetostriction coefficient of magnetostrictive material Terfenol-D changed by the DC magnetic field $H_{d c}$. Compared the tree clamping ways in Table 1 , the averages of the maximum ME coefficients are similar, different within $0.8 \%$. It is believed that the results are effective under the three clamping ways. The averages under the free-stress holding are slightly smaller, because the extra stress from the first two clamping ways is mostly compressive stress. In the equation (3), substitute the material parameters of Terfenol-D and PZT, the coefficient $g_{x}$ is a positive term on the testing direction, so the testing results under the first two clamping ways is some larger than by the free-stress holding way. The standard deviation of the free-stress holding is smallest under the three clamping ways. Because the uncertainty of the extra stress caused by the clamping ways will add a new error sauce of the ME coefficient testing results, the dispersion of the results increases, in other words, the accuracy of the results is reduced. Therefore, the free-stress holder improved the accuracy of the testing results of the measurement system.

Figures $9(\mathrm{a})-(\mathrm{c})$ are the relationships between the ME coefficient and the
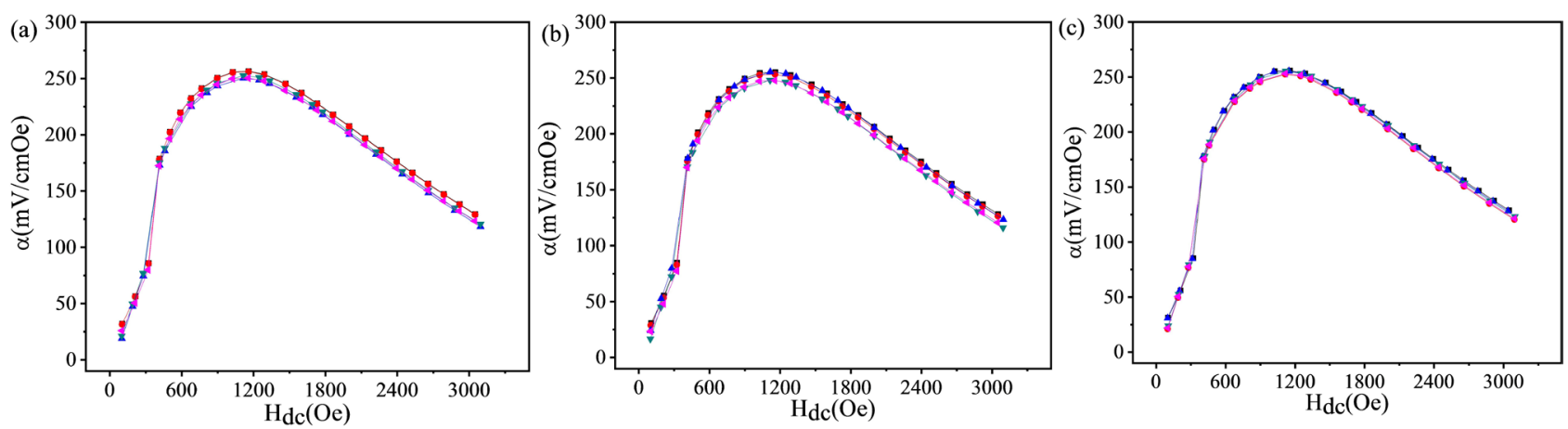

Figure 8. The ME coefficient vs. DC magnetic field at the AC magnetic field of $1 \mathrm{kHz}$, (a) clamping vertically, (b) clamping horizontally and (c) free-stress holding.

Table 1. Average value and Standard deviation of $\left(\alpha_{E}\right)_{\max }$ by three clamping ways.

\begin{tabular}{cccc}
\hline the curve of $\boldsymbol{\alpha}_{E}-H$ & (a) clamping vertically & (b) clamping horizontally & (c) free-stress holding \\
\hline Average value of $\left(\alpha_{E}\right)_{\max }(\mathrm{mV} / \mathrm{cmOe})$ & 255.1 & 255.3 & 255.2 \\
Standard deviation of $\left(\alpha_{E}\right)_{\max }(\mathrm{mV} / \mathrm{cmOe})$ & 2.94 & 4.02 & 1.27 \\
\hline
\end{tabular}



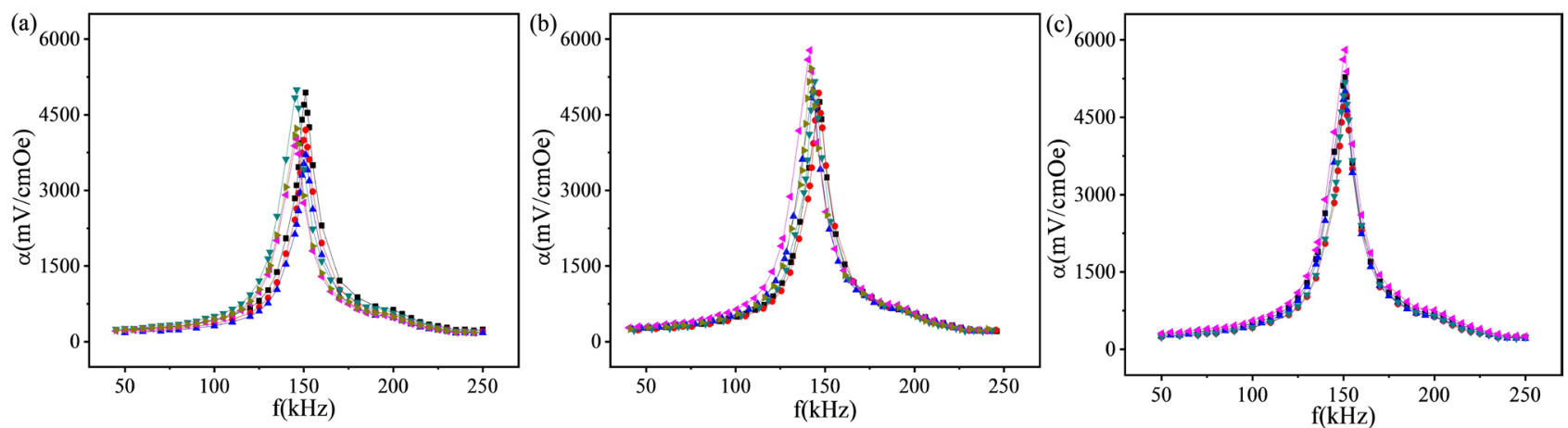

Figure 9. The ME coefficient vs. the frequency of AC magnetic field at DC magnetic field of 600 Oe, (a) clamping vertically, (b) clamping horizontally and (c) free-stress holding.

Table 2. Average value and standard deviation of $\left(\alpha_{E}\right)_{\max }$ by three clamping ways.

\begin{tabular}{cccc}
\hline the curve of $\boldsymbol{\alpha}_{E}-\boldsymbol{f}$ & (a) clamping vertically & (b) clamping horizontally & (c) free-stress holding \\
\hline Average value of $\left(\alpha_{E}\right)_{\max }(\mathrm{mV} / \mathrm{cmOe})$ & 4952.8 & 5175.0 & 5241.2 \\
Standard deviation of $\left(\alpha_{E}\right)_{\max }(\mathrm{mV} / \mathrm{cmOe})$ & 557.2 & 416.6 & 344.1 \\
\hline
\end{tabular}

frequency of the AC magnetic field at DC magnetic field of 600 Oe. Near by the resonant frequency of the sample, there are resonant frequency peaks of ME coefficient, which peak value is hundreds of times larger than the value at away from the resonant frequency, all appeared at $149-151 \mathrm{kHz}$. The averages of the peaks under the three clamping ways are similar and the standard deviation of the free-stress holding is smallest, shown in Table 2. The extra stresses under the clamping ways increase the error sauce and increase the dispersion of the testing results, which results in reducing the accuracy of the results using the measurement system.

\section{Conclusion}

In this paper, a ME coefficient measurement system was designed with a freestress sample holder. Compared with the other clamping ways, the free-stress sample holder reduced the extra stress from the clamping ways, resulting in improving the accuracy of the testing result of the measurement system. Finally, a ME coefficient measurement system was designed including the five modules, DC magnetic field generation module, AC magnetic field generation module, the induced ME voltage detecting module, a sample holder and PC software module, and the performance of the system were as follows: bias magnetic field range is 0 - 4000 Oe; AC magnetic field amplitude is 1 Oe and the frequency is $0.01-300$ $\mathrm{kHz}$; the ME coefficient's accuracy is $0.01 \mathrm{mV} / \mathrm{cm}$.Oe by lock-in amplifier and $0.05 \mathrm{mV} / \mathrm{cm} \cdot$ Oe by the multi-meter.

\section{Acknowledgements}

This paper was funded by the National Natural Science Foundation of China (No: 61271072). 


\section{Conflicts of Interest}

The authors declare no conflicts of interest regarding the publication of this paper.

\section{References}

[1] Bibes, M. and Barthelemy, A. (2008) Multiferroics: Towards a Magnetoelectric Memory. Nature Materials, 7, 425-426. https://doi.org/10.1038/nmat2189

[2] Wei, Y.P. and Gao, C.X. (2016) Four-State Memory Based on a Giant and Non-Volatile Converse Magnetoelectric Effect in FeAl/PIN-PMN-PT Structure. Scientific Reports, 6, Article No. 30002. https://doi.org/10.1038/srep30002

[3] Reermann, J., Zabel, S., Kirchhof, C., Quandt, E., Faupel, F. and Schmidt, G. (2016) Adaptive Readout Schemes for Thin-Film Magnetoelectric Sensors Based on the Delta-E Effect. IEEE Sensors Journal, 16, 4891-4900. https://doi.org/10.1109/JSEN.2016.2553962

[4] Petrie, J., Gray, D. and Viehland, D. (2012) Shifting the Operating Frequency of Magnetoelectric Sensors. Journal of Applied Physics, 111, 07C714.

https://doi.org/10.1063/1.3677840

[5] Cristina, E.C., Ovidiu, G.A., Ioan, D., Mirela, A., Sorin, T., Florin, T. and Liliana, M. (2016) Engineering Magnetoelectric Composites towards Application as Tunable Microwave Filters. Journal of Physics D: Applied Physics, 49, Article ID: 125002. https://doi.org/10.1088/0022-3727/49/12/125002

[6] Zhou, H.M., Lian, J. and Zhu, F.J. (2014) The Lumped Equivalent Circuit Model of the Multi-Passband Tunable Microwave Magnetoelectric Filters. Journal of Applied Physics, 116, Article ID: 063904. https://doi.org/10.1063/1.4892938

[7] Eerenstein, W., Mathur, N.D. and Scott, J.F. (2006) Multiferroic and Magnetoelectric Materials. Nature, 442, 759-765. https://doi.org/10.1038/nature05023

[8] Fiebig, M. (2005) Revival of the Magnetoelectric Effect. Journal of Physics D: Applied Physics, 38, R123. https://doi.org/10.1088/0022-3727/38/8/R01

[9] Suryanarayana, S.V. (1994) Magnetoelectric Interaction Phenomena in Materials. Bulletin of Materials Science, 17, 1259-1270. https://doi.org/10.1007/BF02747225

[10] Mahesh Kumar, M., Srinivas, A., Suryanarayana, S.V., et al. (1998) An Experimental Setup for Dynamic Measurement of Magnetoelectric Effect. Bulletin of Materials Science, 21, 251-255. https://doi.org/10.1007/BF02744978

[11] Lu, J., Pan, D., et al. (2008) Wideband Magnetoelectric Measurement System with the Application of a Virtual Multi-Channel Lock-In Amplifier. Measurement Science and Technology, 19, Article ID: 045702. https://doi.org/10.1088/0957-0233/19/4/045702

[12] Vopson, M.M., Fetisov, Y.K., Caruntu, G. and Srinivasan, G. (2017) Measurement Techniques of the Magneto-Electric Coupling in Multiferroics. Materials, 10, 963. https://doi.org/10.3390/ma10080963

[13] Dong, S. (2004) Characterization of Magnetoelectric Laminate Composites Operated in Longitudinal-Transverse and Transverse-Transverse Modes. Journal of Applied Physics, 95, 2625. https://doi.org/10.1063/1.1644027

[14] Zhao, C.P., Fang, F. and Yang, W. (2010) A Dual-Peak Phenomenon of Magnetoelectric Coupling in Laminated Terfenol-D/PZT/Terfenol-D Composites. Smart Materials and Structures, 19, Article ID: 125004.

https://doi.org/10.1088/0964-1726/19/12/125004 
[15] Thayer, N., Butler, P., et al. (2011) Global Magnetoelectric Measurement System (GMMS). Integrated Ferroelectrics, 131, 173-184. https://doi.org/10.1080/10584587.2011.616442

[16] Gao, C., Hu, B., et al. (2005) Measurement of the Magnetoelectric Coefficient Using a Scanning Evanescent Microwave Microscope. Applied Physics Letters, 87, Article ID: 153505 . https://doi.org/10.1063/1.2093925

[17] Vopsaroiu, M., Stewartet, M., et al. (2008) Experimental Determination of the Magnetoelectric Coupling Coefficient via Piezoelectric Measurements. Measurement Science and Technology, 19, Article ID: 045106. https://doi.org/10.1088/0957-0233/19/4/045106

[18] Ryu, J., Priya, S., et al. (2002) Magnetoelectric Effect in Composites of Magnetostrictive and Piezoelectric Materials. Journal of Electroceramics, 8, 107-119. https://doi.org/10.1023/A:1020599728432

[19] Cheng, J.H., Wang, Y.G., et al. (2014) Resonance Magnetoelectric Effect in $\mathrm{Ni} / \mathrm{Pb}(\mathrm{Zr}, \mathrm{Ti}) \mathrm{O}_{3} /$ Terfenol-D Trilayered Composites with Different Mechanical Boundary Conditions. Applied Physics Letters, 104, Article ID: 252411.

https://doi.org/10.1063/1.4885515 